\title{
Técnica quirúrgica y resultados a corto plazo de la hernioplastía incisional intraperitoneal abierta*
}

\author{
Drs. MARCELO A. BELTRÁN S. ${ }^{1,2}$, LUIS IVÁN JARAMILLO R. ${ }^{1,2}$, CRISTÓBAL LARRAÍN T. ${ }^{1,2}$, \\ PEDRO ERNESTO MÉNDEZ P. ${ }^{1,2}$, JUAN OLIVA S. ${ }^{1,2}$, MARIO A. CONTRERAS G. ${ }^{1,2}$, \\ RODRIGO TAPIA L. ${ }^{1,2}$, VÍCTOR J. CORTÉS F. ${ }^{1,2}$, E.U. KARINA S. CRUCES B. ${ }^{1}$ \\ 1 Servicio de Cirugía, Hospital de La Serena. \\ 2 Escuela de Medicina, Universidad Católica del Norte. \\ La Serena, Chile.
}

\begin{abstract}
Surgical technique and short-term outcomes of open intraperitoneal incisional hernioplasty

Introduction: A review of current literature failed to find any detailed description on the surgical technique of open intraperitoneal mesh hernia repair. The aim of the present study was to describe the open surgical technique used at our institution to repair incisional hernias with intraperitoneal composite prosthesis, and the short-term outcomes including recurrence, complications and patient satisfaction with the procedure. Patients and Methods: A transversal, observational and descriptive report on open intraperitoneal hernioplasty was designed. Short-term outcomes were assessed at a transversal cut out point at a 12-month interview during the follow-up. The interview included a physical exam and a specific questionnaire inquiring over the patient's satisfaction with the procedure. Results: According to the inguinal ring location, most hernias originated between the xiphoid appendix and the umbilicus (64\%). Mean surgical time was 2.6 hours. One or more complications presented in 11 patients $(19 \%)$. One year after surgery there was not any recurrence. According to the patient' satisfaction questionnaire final score, 17 patients $(29 \%)$ reported an excellent satisfaction with the procedure, 33 patients $(57 \%)$ reported a very good satisfaction and 8 patients $(14 \%)$ a good satisfaction. Conclusions: Intraperitoneal hernioplasty constitutes a secure option to repair incisional hernias. At shortterm follow-up there were no recurrences.
\end{abstract}

Key words: Incisional hernia, intraperitoneal hernioplasty, composite mesh.

\section{Resumen}

Introducción: No se encuentra en la literatura ninguna descripción detallada de la técnica para instalación de prótesis intraperitoneales por vía abierta. El objetivo del presente estudio es describir la técnica que utilizamos en nuestra institución y los resultados a corto plazo de estas operaciones: recurrencia, com-

*Recibido el 20 de noviembre de 2011 y aceptado para publicación el 31 de enero de 2012.

Los autores no declaran conflictos de interés.

Correspondencia: Dr. Marcelo A. Beltrán S. Casilla 912, La Serena, Chile. beltran.0608@gmail.com 
plicaciones y satisfacción del usuario con el procedimiento. Pacientes y Método: El presente es un reporte transversal, observacional y descriptivo de la técnica de la hernioplastía intraperitoneal abierta y de los resultados iniciales de esta técnica tomando como punto de corte transversal el control de los 12 meses de seguimiento. El control incluyó una entrevista, examen físico y un cuestionario específico sobre satisfacción con el procedimiento. Resultados: De acuerdo a la localización del anillo herniario, la mayoría de las hernias se originaron entre la cicatriz umbilical y el apéndice xifoides $(60,4 \%)$. El tiempo quirúrgico promedio fue 2,6 horas. Se presentaron una o más complicaciones en 11 pacientes (19\%). Un año después de la cirugía no se encontró ninguna recurrencia. De acuerdo a la puntuación final del cuestionario de satisfacción usuaria, 17 pacientes $(29 \%)$ reportaron una satisfacción excelente con el procedimiento, 33 pacientes (57\%) una satisfacción muy buena y 8 pacientes (14\%) una satisfacción buena. Conclusiones: La hernioplastía intraperitoneal constituye una opción segura para la reparación de hernias incisionales. En el seguimiento a corto plazo no presenta recurrencias.

Palabras clave: Hernia incisional, hernioplastía intraperitoneal, malla compuesta.

\section{Introducción}

El concepto de la hernioplastía intraperitoneal se origina durante el desarrollo de las técnicas laparoscópicas para reparación de hernias incisionales. La hernioplastía intraperitoneal laparoscópica constituye una opción segura para el tratamiento de hernias incisionales de la pared abdominal, asociada a una baja tasa de recurrencia que alcanza a $6,25 \%$ a largo plazo $^{1}$. La instalación de prótesis no-reabsorbibles dentro de la cavidad abdominal se asocia a la formación de adherencias, obstrucción intestinal y fistulas enterocutáneas, razones por las cuales se han desarrollado prótesis compuestas que evitarían estas complicaciones $^{2-5}$. En nuestra institución utilizamos la prótesis Proceed ${ }^{\mathrm{TM}}$ desde agosto del año 2008 para la reparación intraperitoneal por vía laparoscópica o abierta de hernias incisionales. La prótesis Proceed ${ }^{\mathrm{TM}}$ está compuesta por una malla liviana de polipropileno $\left(44 \mathrm{~g} / \mathrm{m}^{2}\right)$ recubierta de polidioxanona reabsorbible y celulosa oxidada regenerada (Ethicon, Inc. Somerville, NJ, USA). Las técnicas de instalación laparoscópica de prótesis intraperitoneales se encuentran ampliamente descritas ${ }^{6-8}$. Sin embargo, no encontramos en la literatura ninguna descripción detallada de la técnica para instalación de prótesis intraperitoneales por vía abierta. El objetivo del presente estudio es describir la técnica que utilizamos en nuestra institución para instalación de prótesis intraperitoneales por vía abierta y los resultados a corto plazo de estas operaciones: recurrencia, complicaciones y satisfacción del usuario con el procedimiento.

\section{Pacientes y Método}

\section{Diseño del estudio}

El presente es un reporte transversal, observacional y descriptivo de la técnica de la hernioplastía intraperitoneal abierta (HIPRA) y de sus resultados iniciales en nuestra institución tomando como punto de corte transversal el control de los 12 meses de seguimiento. Entre agosto de 2008 y julio de 2009 se operaron 58 pacientes por hernia incisional con la técnica HIPRA. Las indicaciones de HIPRA fueron: hernias incisionales con pérdida de domicilio, anillo herniario con longitud y ancho mayor a $10 \mathrm{~cm}$ y la falta de insumos laparoscópicos. Todos los pacientes fueron citados a un control de corte transversal 12 meses después de la cirugía. El control incluyó la entrevista, examen físico y un cuestionario específico sobre satisfacción con el procedimiento para pacientes operados por hernia incisional, modificado de una versión previamente establecida para el seguimiento de pacientes con hernia inguinal ${ }^{9}$. Se reporta el tamaño de malla utilizado, el tiempo quirúrgico, el uso de drenajes, la estadía hospitalaria, las complicaciones postoperatorias, recurrencia y los resultados del cuestionario sobre satisfacción con el procedimiento.

\section{Variables y definiciones}

Se estudió el Índice de Masa Corporal (IMC) y su influencia en los resultados de la cirugía. Los pacientes fueron divididos de acuerdo al IMC: Normal 19-24,9 Kg/m²; Sobrepeso 25-29,9 Kg/m²; Obesidad 30-34,9 Kg/m² y Obesidad mórbida $>35$ $\mathrm{Kg} / \mathrm{m}^{2}$ asociada a una o más patologías sistémicas. La hernia se clasificó de acuerdo a la clasificación de la European Hernia Society ${ }^{10}$, que toma en cuenta la localización y el tamaño del anillo herniario (Tabla 1). Se definió como hernia incisional a cualquier orificio de la pared abdominal con o sin saco herniario en el área de una herida postoperatoria, perceptible o palpable durante el examen físico o por medios radiológicos ${ }^{10}$. Se definió como hernia incisional aquella hernia no sometida a ningún procedimiento previo para su reparación y como hernia incisional recurrente aquella hernia que se presenta después de un procedimiento realizado para reparar una hernia incisional $^{10,11}$. 
M. A. BELTRÁN S. y cols.

Tabla 1. Clasificación de la hernia incisional (European Hernia Society, 2009) $^{10}$

\begin{tabular}{|c|c|c|c|}
\hline & \multicolumn{2}{|c|}{ Localización } & Nombre \\
\hline \multirow[t]{5}{*}{ Línea media } & \multicolumn{2}{|c|}{ Subxifoidea } & M1 \\
\hline & \multicolumn{2}{|c|}{ Epigástrica } & M2 \\
\hline & \multicolumn{2}{|c|}{ Umbilical } & M3 \\
\hline & \multicolumn{2}{|c|}{ Infraumbilical } & M4 \\
\hline & \multicolumn{2}{|c|}{ Suprapúbica } & M5 \\
\hline \multirow[t]{4}{*}{ Lateral } & \multicolumn{2}{|c|}{ Subcostal } & $\mathrm{L} 1$ \\
\hline & \multicolumn{2}{|l|}{ Flanco } & L2 \\
\hline & \multicolumn{2}{|l|}{ Ilicaca } & L3 \\
\hline & \multicolumn{2}{|c|}{ Lumbar } & L4 \\
\hline Hernia recurrente & \multicolumn{2}{|l|}{ Sí } & No \\
\hline \multirow{2}{*}{$\begin{array}{ll}\text { Longitud: } & \mathrm{cm} \\
\text { Ancho }(\mathrm{W}) & \end{array}$} & \multicolumn{2}{|l|}{ Ancho: } & \\
\hline & $\begin{array}{l}\text { W1: } \\
<4 \mathrm{~cm}\end{array}$ & $\begin{array}{c}\text { W2: } \\
4-10 \mathrm{~cm}\end{array}$ & $\begin{array}{c}\text { W3: } \\
>10 \mathrm{~cm}\end{array}$ \\
\hline
\end{tabular}

\section{Puntuación para el reporte de la satisfacción con el procedimiento para pacientes operados electivamente por hernia incisional (PRSP)}

Una puntuación similar ha sido previamente aplicada en pacientes operados por hernia inguinal ${ }^{9,11,16}$, sobre la base de esta experiencia, se desarrolló este cuestionario. El reporte de los resultados de la cirugía herniaria comprende no sólo los resultados quirúrgicos, sino también la percepción propia del paciente respecto al procedimiento y sus complicaciones ${ }^{17}$; por estas razones, consideramos que esta puntuación es útil para el reporte de nuestros resultados. Se formulan 3 preguntas con opciones de respuesta cerrada y 2 preguntas de respuesta abierta, una de las cuales no se considera para la suma de los puntos, sino sólo como informativa. La pregunta 1 inquiere directamente sobre el sentimiento general del paciente respecto a su operación. La pregunta 2 evidencia la percepción del paciente respecto a la mejoría en su calidad de vida después de la cirugía. La pregunta 3 evidencia indirectamente la percepción del paciente sobre su experiencia con la cirugía, una respuesta positiva evidencia una buena percepción sobre el procedimiento y una respuesta negativa evidencia una mala percepción. La pregunta 4 se relaciona con la pregunta 3 y requiere una respuesta abierta, esta pregunta es sólo informativa. La pregunta 5 permite al paciente calificar el procedimiento de acuerdo a una escala visual analógica en la que 10 es el puntaje máximo y 0 el mínimo. La suma de las respuestas permite elaborar una escala de puntaje que nos permite objetivar la satisfacción del usuario (Figura 1).

$$
\begin{aligned}
& \text { 1) ¿Cómo se siente Ud., respecto la cirugía de su hernia? } \\
& \text { 1. Peor que antes: } \\
& \text { 2. Mal: } \\
& \text { 3. Igual que antes: - } \\
& \text { 4. Bien: } \\
& \text { 5. Muy bien: - } \\
& \text { 6. Excelente: - } \\
& \text { 2) ¿Ud., siente que esta cirugía ha mejorado su calidad } \\
& \text { de vida? } \\
& \text { 1. Sí: } \\
& \text { 2. No: - } \\
& \text { 3) De ser necesario Ud., se operaría de esta misma cirugía } \\
& \text { nuevamente? } \\
& \text { 1. Sí: - } \\
& \text { 2. No: - } \\
& \text { 4) ¿Por qué? }
\end{aligned}
$$

5) Entre 1 y 10 puntos, ¿cómo calificaría Ud., su experiencia con esta operación? (Utilizar escala visual analógica)

\section{PUNTUACIÓN FINAL}

1. Satisfacción excelente:

2. Satisfacción muy buena:

16 a 18 puntos

3. Satisfacción buena:

13 a 15 puntos

- El puntaje final se calcula mediante la suma de las preguntas: $1+2+3+5$

- El valor de las preguntas es el siguiente:

Pregunta 1: 1 a 6 puntos de acuerdo a la respuesta Preguntas 2 y 3: 1 punto cada una si la respuesta es "Sí" - Si la respuesta es "No" el valor es 0

Pregunta 4: No tiene puntaje - Esta es una pregunta con intenciones exclusivamente informativas Pregunta 5: 0 a 10 puntos de acuerdo a la respuesta

Figura 1. Puntuación para el reporte de la satisfacción con el procedimiento para pacientes operados electivamente por hernia incisional (PRSP).

\section{Técnica quirúrgica}

Los siguientes pasos iniciales son similares a los previamente descritos ${ }^{12-15}$ :

1. Resección de la cicatriz antigua (Figura 2A).

2. Disección del saco herniario hasta delimitar el anillo herniario (Figura 2B).

3. Tallado de colgajos de piel liberando el tejido adiposo subcutáneo hasta la aponeurosis a una distancia de $6 \mathrm{~cm}$ desde el borde del anillo herniario.

4. Apertura y resección del saco herniario, liberando el anillo herniario de todas las adherencias peritoneales (Figura 2C). 


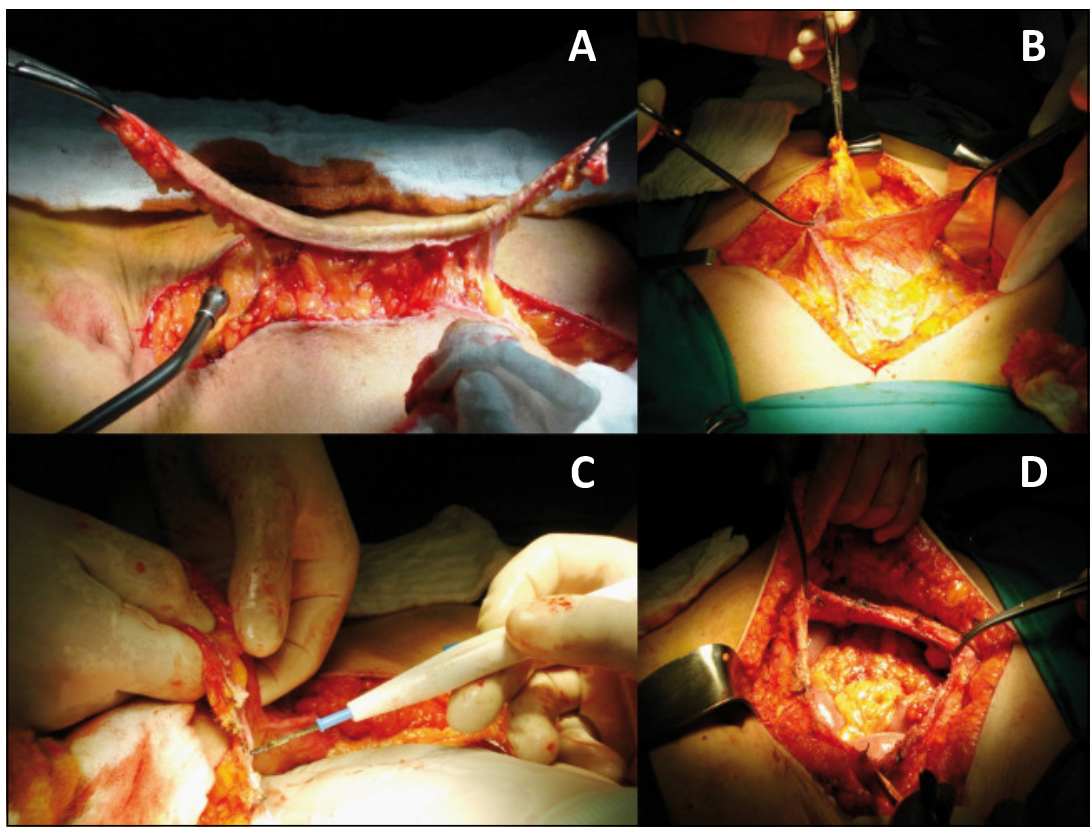

Figura 2. Disección inicial de la hernia incisional.
5. Exploración de la cavidad abdominal y liberación de las asas intestinales de sus adherencias al anillo herniario (Figura 2D). Es necesario liberar de toda adherencia, por lo menos $5 \mathrm{~cm}$ de pared abdominal interna para poder fijar la malla apropiadamente. En muchas ocasiones es necesario liberar el ligamento redondo del hígado y el ligamento falciforme.

Se han publicado estudios sobre la instalación de la malla intraperitoneal por vía abierta ${ }^{18}$, sin embargo, no existe una descripción detallada de esta técnica. La siguiente es la técnica que utilizamos:

1. Se pasan puntos separados de polipropileno $2 / 0$ a través de la aponeurosis hacia dentro de la cavidad abdominal y se dejan tomados en "U" con una pinza Kelly (Figura 3). Los puntos laterales deben pasar por el lado lateral de la vaina de los músculos rectos anteriores del abdomen.

2. Se repite la maniobra alrededor de todo el anillo herniario hasta formar una "corona" de puntos (Figuras 4A y 4B).

3. A continuación se pasan los puntos cerca del borde de la malla en "U"; entrando por la parte superior no absorbible hacia la parte inferior cubierta de celulosa absorbible que estará en contacto con los intestinos y nuevamente a través de la aponeurosis desde dentro de la cavidad abdominal hacia fuera cerca del punto de entrada de cada punto hasta completar la corona. La malla desciende en la medida que se van pasando los puntos (Figura 5A).

4. Se anudan los puntos y la malla queda en posición intraperitoneal absolutamente sin tensión. El anillo herniario se fija a la malla con una sutura continua de polipropileno 2/0 (Figura 5B). En algunos casos es posible cerrar parcialmente la aponeurosis sobre la malla.

6. Se dejan drenajes aspirativos.

\section{Estadística}

Las variables dicotómicas y categóricas se presentan como frecuencias y porcentajes y se analizaron con el test $\chi^{2}$. Las variables continuas se presentan como mediana o promedio, desviación estándar; valores mínimos y máximos. Se utilizó el test de Pearson para evidenciar correlaciones entre las siguientes variables: edad, IMC, hernia recurren-

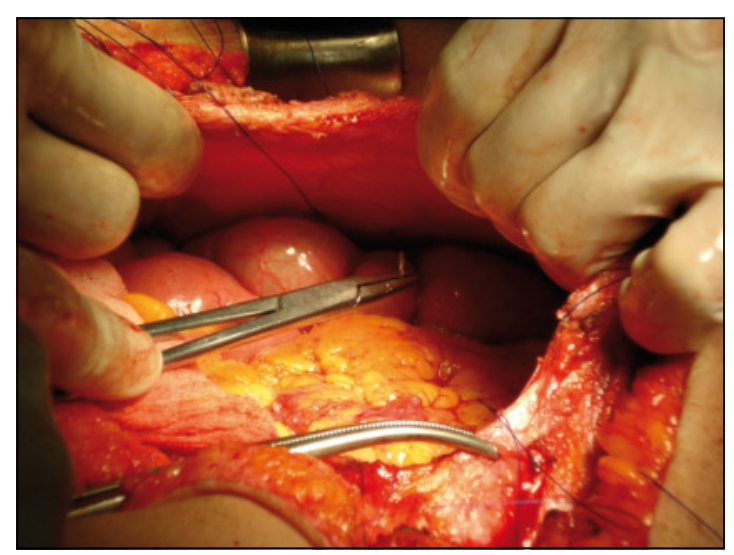

Figura 3. Paso de los puntos transfasciales en "U" (vista intra-abdominal). 


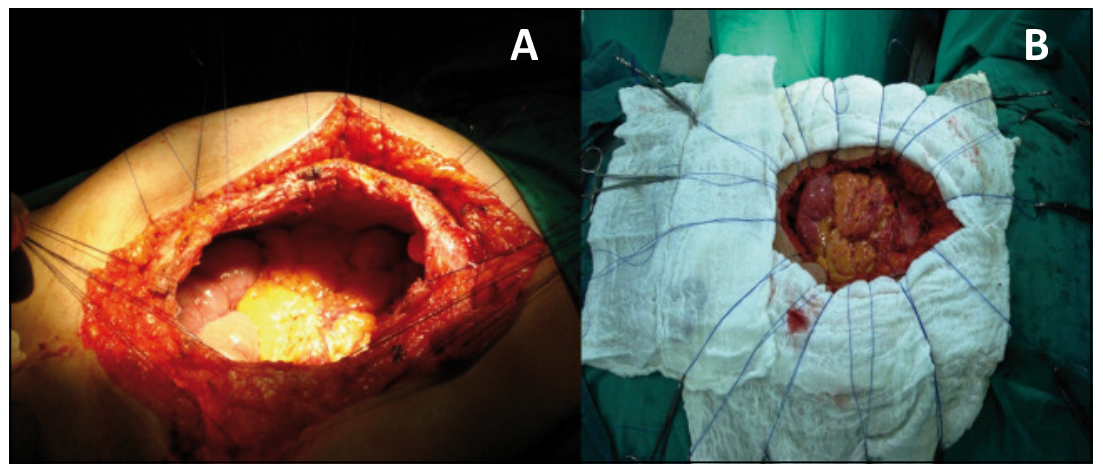

Figura 4. "Corona" de puntos transfasciales concluida.

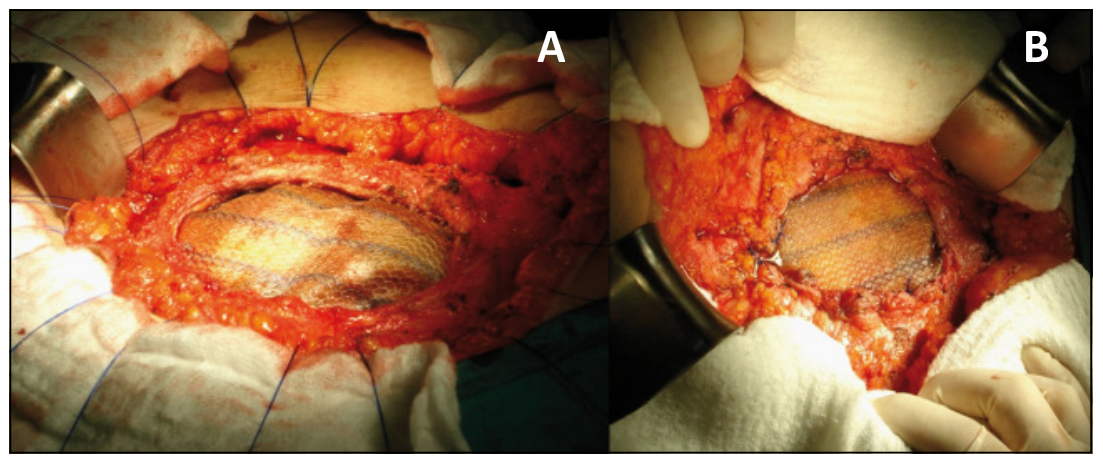

Figura 5. Fijación de la malla.

te, longitud de la hernia, tiempo quirúrgico, tiempo de estadía hospitalaria y puntaje final del cuestionario. La base de datos se construyó y analizó con el software SPSS versión 11.0.

\section{Resultados}

La mayoría de los pacientes fueron de sexo femenino (49 mujeres $84,5 \%$ versus 9 varones $15,5 \%$, $\mathrm{p}<0,0001$ ), la mediana de edad fue 55 años, siendo la mayoría pacientes mayores de 50 años (39 casos, $67 \%$ ). La mediana del IMC fue $27,5 \mathrm{~kg} / \mathrm{m}^{2}$, encontrándose la mayoría de los pacientes con sobrepeso u obesidad (34 casos, 58\%) (Tabla 2). De acuerdo a la localización del anillo herniario, la mayoría de las hernias se originaron entre la cicatriz umbilical y el apéndice xifoides (41 hernias 60\% supraumbilicales versus 17 hernias $40 \%$ en otras localizaciones, $\mathrm{p}=0,006$ ). La mediana de la longitud del anillo herniario fue $14 \mathrm{~cm}$, siendo más frecuentes aquellas hernias con una longitud entre 11 y $20 \mathrm{~cm}$ (26 casos $45 \%, \mathrm{p}<0,0001)$. La mediana de anchura del anillo herniario fue $10,7 \mathrm{~cm}$, siendo más frecuentes aquellos anillos con una anchura entre 4 y $10 \mathrm{~cm}$ (28 casos, 48\%, $\mathrm{p}<0,0001)$. Las hernias incisionales recurrentes fueron menos frecuentes ( 24 casos $41 \%$
Tabla 2. Información general

\begin{tabular}{|lcc|}
\hline Edad: $55 \pm 17,2$ años $(19-79)$ & $\mathbf{n}$ & $\mathbf{\%}$ \\
$19-29$ & 7 & 12 \\
$30-49$ & 12 & 21 \\
$50-69$ & 25 & 43 \\
$>70$ & 14 & 24 \\
Género & $\mathbf{n}$ & $\mathbf{\%}$ \\
Femenino & 49 & 84,5 \\
Masculino & 9 & 15,5 \\
Índice de masa corporal: & $\mathbf{n}$ & $\mathbf{\%}$ \\
$27,5 \pm 6,9 \mathrm{~kg} / \mathrm{m}^{2}(15-45)$ & & \\
$15-20$ & 5 & 9 \\
$21-25$ & 15 & 26 \\
$26-30$ & 10 & 17 \\
$31-35$ & 10 & 17 \\
$36-40$ & 12 & 21 \\
$>41$ & 2 & 3 \\
\hline
\end{tabular}

versus 34 casos $59 \%, \mathrm{p}=0,016)$ al igual que las hernias con anillo único ( 28 casos $48 \%$ versus 30 casos $52 \%, \mathrm{p}=0,146$ ) (Tabla 3). El tamaño de malla utilizado dependió del tamaño del anillo herniario. El tiempo quirúrgico promedio fue 2,6 horas. En la mayoría de los casos se utilizaron drenajes (44 pacien- 
TÉCNICA QUIRÚRGICA Y RESULTADOS A CORTO PLAZO DE LA HERNIOPLASTÍA INCISIONAL INTRAPERITONEAL...

Tabla 3. Características de las hernias

\begin{tabular}{|lrc|}
\hline Localización & $\mathbf{n}$ & $\mathbf{\%}$ \\
M1: Subxifoidea & 6 & 10 \\
M2: Epigástrica & 31 & 53 \\
M3: Umbilical & 4 & 7 \\
M4: Infraumbilical & 12 & 21 \\
M5: Suprapúbica & 5 & 9 \\
Total & 58 & 100 \\
Longitud: $14 \pm 6,7 \mathrm{~cm}(5,5-28,5)$ & $\mathbf{n}$ & $\mathbf{\%}$ \\
5 - 10 & 18 & 31 \\
11 - 20 & 26 & 45 \\
$>$ 21 & 14 & 24 \\
Ancho (W): $10,7 \pm 5,4 \mathrm{~cm}(3-27)$ & $\mathbf{n}$ & $\mathbf{\%}$ \\
W1: $<4$ & 19 & 33 \\
W2: $4-10$ & 28 & 48 \\
W3: $>10$ & 11 & 19 \\
Hernia incisional & 34 & 59 \\
Hernia recurrente & 24 & 41 \\
Anillo fenestrado & 30 & 52 \\
Anillo único & 28 & 48 \\
& &
\end{tabular}

tes $76 \%, \mathrm{p}=0,007)$ (Tabla 4). La mediana de estadía hospitalaria fue 5 días, la mayoría de los pacientes permanecieron hospitalizados menos de 5 días (39 casos $67 \%, \mathrm{p}=0,024$ ) (Tabla 5). Se presentaron una o más complicaciones en 11 pacientes (19\%) lo que prolongó la estadía hospitalaria en 6 pacientes por 11 días o más. La complicación más frecuente fue la infección de sitio quirúrgico superficial en 6 pacientes (11\%), la complicación más grave fue la necrosis de la piel alrededor de la incisión en un paciente, la cual fue resuelta mediante el sistema de aspiración cerrada (Tabla 6). Un año después de la cirugía no se encontró ninguna recurrencia en toda la cohorte. A la primera pregunta del cuestionario PRSP la mayoría de los pacientes (33 casos, 57\%) respondieron que se sentían muy bien respecto a su cirugía; todos los pacientes $(100 \%)$ sintieron que la cirugía ha mejorado su calidad de vida; 32 pacientes $(55 \%)$ se operarían nuevamente con la misma técnica, de ellos 18 pacientes lo harían para recuperar su salud y 10 pacientes sólo en caso necesario. La mayoría de los pacientes ( 30 casos, $52 \%$ ) calificaron el procedimiento con 8 puntos y 14 pacientes (24\%) con 9 puntos (Tabla 7). Tres pacientes $(5 \%)$ obtuvieron el puntaje máximo del PRSP (Figura 6). De acuerdo a la puntuación final 17 pacientes (29\%) reportaron una satisfacción excelente con el procedimiento, 33 pacientes $(57 \%)$ una satisfacción muy buena y 8 pacientes $(14 \%)$ una satisfacción buena (Figura 7). El test de Pearson demostró las si-
Tabla 4. Características de la cirugía

\begin{tabular}{|lcc|}
\hline Tamaño de la malla (cm) & n & $\mathbf{\%}$ \\
$15 \times 15 \mathrm{~cm}$ Cuadrada & 22 & 38 \\
15 x $20 \mathrm{~cm}$ Oval & 22 & 38 \\
30,5 x $30,5 \mathrm{~cm}$ Cuadrada & 14 & 24 \\
Tiempo quirúrgico (h) & $\mathbf{n}$ & $\mathbf{\%}$ \\
$2 \mathrm{~h}$ & 35 & 60 \\
$3 \mathrm{~h}$ & 12 & 21 \\
$4 \mathrm{~h}$ & 8 & 14 \\
$5 \mathrm{~h}$ & 3 & 5 \\
Promedio: $2,6 \pm 0,9 \mathrm{~h}(2-5 \mathrm{~h})$ & & \\
Drenajes & $\mathbf{n}$ & $\mathbf{\%}$ \\
Sí & 44 & 76 \\
No & 14 & 24 \\
1 drenaje & 6 & 10 \\
2 drenajes & 35 & 60 \\
3 drenajes & 2 & 3 \\
4 drenajes & 1 & 2 \\
\hline
\end{tabular}

Tabla 5. Estadía hospitalaria

\begin{tabular}{|lcc|}
\hline Tiempo de estadía hospitalaria (días) & n & $\%$ \\
\hline $2-5$ & 39 & 67 \\
$6-10$ & 13 & 22 \\
$>11$ & 6 & 11 \\
\hline Promedio: $5 \pm 4,9$ días $(2-21$ días $)$ & & \\
\hline
\end{tabular}

Tabla 6. Complicaciones

\begin{tabular}{|lcc|}
\hline Complicaciones & n & \% \\
Infección de sitio quirúrgico superficial & 6 & 11 \\
Seroma persistente & 5 & 9 \\
Dolor prolongado en el sitio quirúrgico & 3 & 5 \\
Íleo postoperatorio prolongado & 2 & 3 \\
Hematoma de sitio quirúrgico & 1 & 2 \\
Dehiscencia de la sutura de la piel & 1 & 2 \\
Necrosis de piel & 1 & 2 \\
Total de pacientes con complicaciones & 11 & $19^{*}$ \\
\hline
\end{tabular}

*En un mismo paciente se presentaron una o más complicaciones.

guientes correlaciones positivas: cuando la hernia es recurrente el tiempo de estadía hospitalaria es mayor $(\mathrm{p}=0,033)$; el puntaje del PRSP es menor cuando el IMC es mayor $(\mathrm{p}=0,023)$; la longitud del anillo herniario es mayor en pacientes con IMC elevado $(\mathrm{p}=0,004)$; los pacientes con IMC elevado tienen 
M. A. BELTRÁN S. y cols.

Tabla 7. Resultados del cuestionario PRSP

\begin{tabular}{|lrr|}
\hline Pregunta & n & $\%$ \\
1. ¿Cómo se siente Ud. respecto & & \\
a su cirugía de hernia? & & \\
Bien & 18 & 31 \\
Muy Bien & 33 & 57 \\
Excelente & 7 & 12 \\
2. ¿Ud. siente que esta cirugía ha & & \\
mejorado su calidad de vida? & & \\
Sí & 58 & 100 \\
No & - & - \\
3. ¿De ser necesario Ud. se operaría & & \\
de esta misma cirugía nuevamente? & & \\
Sí & 32 & 55 \\
No & 26 & 45 \\
4. ¿Por qué? & & \\
Sí: recuperar la salud & 18 & 31 \\
Sí: en caso necesario & 10 & 17 \\
Sí: satisfacción con la cirugía & 1 & 2 \\
Sí: confianza en el cirujano & 3 & 5 \\
No: muy anciano para otra cirugía & 1 & 2 \\
No: demasiadas cirugías previas & 21 & 36 \\
No: por las complicaciones & 4 & 7 \\
sufridas con la cirugía & & \\
5. Entre 1 y 10 puntos ¿cómo calificaría & & \\
su experiencia con esta operación? & & \\
7 puntos & 6 & 10 \\
8 puntos & 30 & 52 \\
9 puntos & 14 & 24 \\
10 puntos & 8 & 14 \\
\hline
\end{tabular}

un mayor tiempo de estadía hospitalaria $(\mathrm{p}=0,007)$; la longitud mayor del anillo herniario se asocia a un tiempo quirúrgico mayor $(\mathrm{p}<0,0001)$ y a un mayor tiempo de estadía hospitalaria $(\mathrm{p}<0,0001)$.

\section{Discusión}

Habitualmente, las hernias incisionales se presentan en pacientes de género femenino, en la quinta o sexta década de la vida, con sobrepeso u obesi$\operatorname{dad}^{1,15,18-22}$, características presentes en esta serie. La obesidad y la edad avanzada son factores de riesgo para el desarrollo de hernias incisionales ${ }^{15,18-22}$, constituyendo además en sí mismas factores de riesgo asociados a morbilidad y mortalidad para la resolución quirúrgica de estas hernias ${ }^{15,18-22}$. Las grandes hernias incisionales se originan en un anillo herniario que se va dilatando en el tiempo y con el aumento

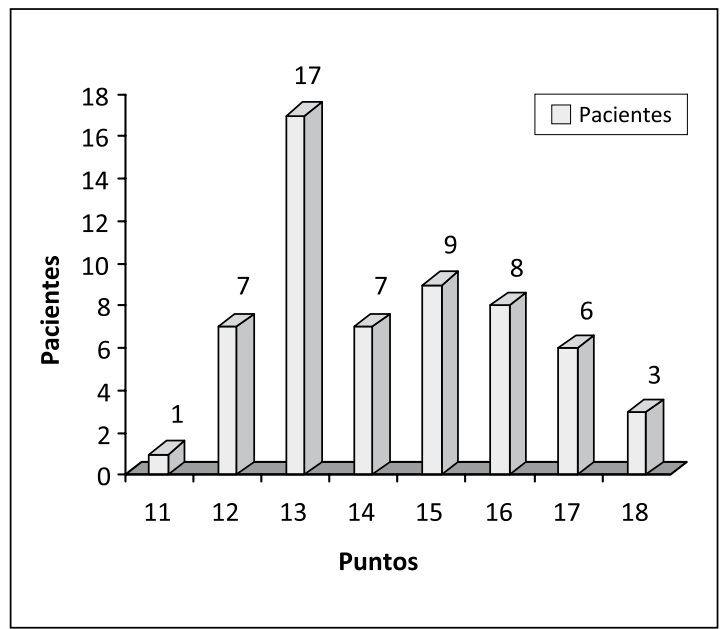

Figura 6. Puntaje del PRSP.

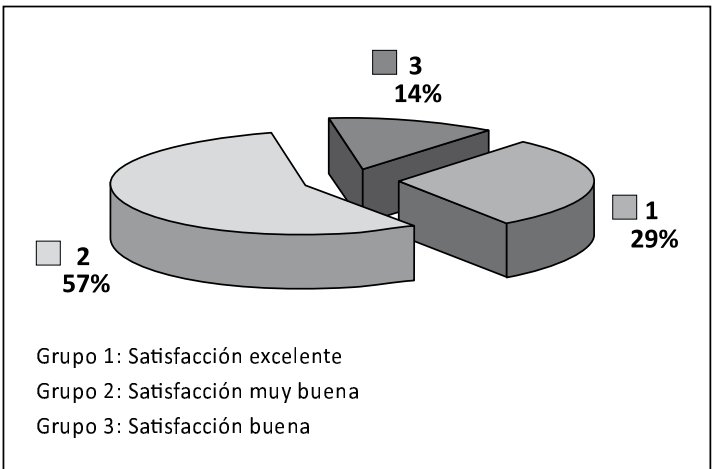

Figura 7. Puntuación final del PRSP.

de la presión intra-abdominal, la cual además está asociada a obesidad y relajación de la musculatura abdominal $^{16,18-22}$, concomitantemente el saco herniario va creciendo ${ }^{16,18-22}$. La reparación de estas grandes hernias incisionales ha sido motivo de innumerables estudios buscando las técnicas más adecuadas, con menor morbilidad y principalmente con menor o ninguna recurrencia. Este ideal no ha sido aún alcanzado y la literatura actual nos demuestra que la morbilidad de la reparación abierta de la hernia incisional varía entre $30 \%$ y $49 \%$, debido principalmente a seroma e infección ${ }^{13,19,22}$, con una tasa de recurrencia que fluctúa entre $2 \%$ y $36 \%{ }^{1,12-14,18,19,22}$; y mortalidad postoperatoria entre $2 \%$ y $13 \%{ }^{18,19}$. Nuestra serie tiene una tasa de complicaciones mucho menor a la publicada, con una tendencia similar al tipo de complicaciones habituales; seroma e infección, sin mortalidad asociada.

Diferentes técnicas de reparación abierta han sido 
desarrolladas, desde la simple sutura de la hernia hasta las complejas cirugías retromusculares que aprovechan la presión intra-abdominal para fijar la malla propuestas por Stoppa y Rives ${ }^{12,15-19}$. La hernioplastía laparoscópica es una solución bastante apropiada, con una tasa de recurrencia a largo plazo que varía entre $3 \%$ y $9 \%$, con una curva de aprendizaje asociada a morbilidad severa $(10,5 \%)$, principalmente por lesiones intestinales $(1 \%$ a $6 \%)$ y a mortalidad $(0,5 \%)^{1,6}$; y habitualmente limitada por razones técnicas a hernias incisionales pequeñas ${ }^{20,23}$. La técnica que presentamos (HIPRA) es bastante simple de reproducir y los resultados en términos de recurrencia son excelentes a corto plazo $(0 \%$ a 12 meses). Sin embargo, se asocia a una morbilidad no despreciable, principalmente debido a seroma, ya que la malla queda expuesta directamente al tejido graso subcutáneo y a la extensa disección de los colgajos de piel necesarios para la fijación de la malla con los puntos transfasciales. La recurrencia de la hernia incisional operada por vía abierta es precoz y se produce dentro de los 2 primeros meses después de la cirugía ${ }^{24,25}$, por esta razón, es que la recurrencia de $0 \%$ a los 12 meses en esta serie, aun cuando representa un seguimiento a muy corto plazo, es muy significativa. El seguimiento a largo plazo demuestra un aumento progresivo y continuo de las recurrencias ${ }^{13-15}$, posiblemente debido al diagnóstico tardío de estas recurrencias o a las manifestaciones clínicas tardías de pequeñas recurrencias iniciales que aumentan de tamaño con el tiempo ${ }^{14}$. Los resultados del cuestionario sobre satisfacción con el procedimiento (PRSP) demuestran que el usuario se encuentra muy o excelentemente satisfecho con este procedimiento a pesar de la morbilidad, lo más notorio es que todos los pacientes sienten que la cirugía mejoró su calidad de vida, percepción que es importante debido a que estos pacientes sufren física y emocionalmente por la hernia. La importancia de este cuestionario es su aplicabilidad durante la entrevista de seguimiento; debido a que habitualmente los estudios que reportan resultados de satisfacción usuaria refieren encuestas telefónicas y no describen el tipo de cuestionario utilizado ${ }^{12,14,15,19,20-22}$.

Finalmente, las correlaciones encontradas demuestran objetivamente que las hernias recurrentes cuando se operan se asocian a una mayor estadía hospitalaria: Un IMC elevado se asocia a hernias más grandes y a una estadía hospitalaria mayor, debido no sólo a una hernia más grande con mayor tiempo quirúrgico sino también a que las complicaciones como el seroma o la dehiscencia de la sutura de la piel o la necrosis de los bordes de la herida se presentaron con mayor frecuencia en estos pacientes. Finalmente, las hernias más grandes requieren tiempos quirúrgicos y de hospitalización mayores.

\section{Conclusiones}

La hernioplastía intraperitoneal con malla compuesta constituye una opción segura para la reparación de hernias incisionales y se asocia a una morbilidad relacionada con las características propias de los pacientes y de la técnica. En el seguimiento a corto plazo, no presenta recurrencias.

\section{Referencias}

1. Moreno-Egea A, Castillo JA, Girela E, Aguayo-Albasini JL. Long-term results of laparoscopic repair of incisional hernias using an intraperitoneal composite mesh. Surg Endosc. 2010;24:359-65.

2. Aguayo-Albasini JL, Moreno-Egea A, Torralba-Martínez JA. The labyrinth of composite prostheses in ventral hernias. Cir Esp. 2009;86:139-46.

3. Burger JW, Halm JA, Wijsmuller AR, ten Raa S, Jeekel $J$. Evaluation of new prosthetic meshes for ventral hernia repair. Surg Endosc. 2006;20:1320-5.

4. Harrell AG, Novitsky YW, Cristiano JA, Gersin JA, Norton HJ, Kercher KW, et al. Prospective histologic evaluation of intra-abdominal prosthetics four months after implantation in a rabbit model. Surg Endosc. 2007;21:1170-4.

5. Schug-Pass C, Sommerer F, Tannapfel A, Lippert H, Köckerling $\mathrm{F}$. The use of composite meshes in laparoscopic repair of abdominal wall hernias. Are there differences in biocompatibility?-Experimental results obtained in a laparoscopic porcine model. Surg Endosc. 2009;23:487-95.

6. Novitsky YW, Paton BL, Heniford BT. Laparoscopic ventral hernia repair. Op Tech Gen Surg. 2006;8:4-9.

7. Gersin KS. Laparoscopic incisional hernia repair. Op Tech Gen Surg. 2004;6:189-99.

8. Curcillo PG. Laparoscopic ventral hernia repair-Simplified with a self-expanding mesh system (A one-suture technique). Op Tech Gen Surg. 2004;6:200-8.

9. Beltrán MA, Burgos C, Almonacid J, Tapia TF, Vicencio A, Danilova T, et al. Long-term follow-up of tension-free Lichtenstein hernioplasty: Application of a qualitative-and-quantitative measurement instrument. Hernia 2005;9:368-74.

10. Muysoms FE, Miserez M, Berrevoet F, Campanelli G, Champault GG, Chelala E, et al. Classification of primary and incisional abdominal wall hernias. Hernia 2010;13:407-14.

11. Beltrán MA, Cruces KS. Outcomes of Lichtenstein hernioplasty for primary and recurrent inguinal hernia. World J Surg. 2006;30:2281-87.

12. Schumpelick V, Klinge U, Junge K, Stumpf M. Incisional abdominal hernia: The open mesh repair. Langenbecks Arch Surg. 2004;389:1-5.

13. Klinge U, Conze J, Krones CJ, Schumpelick V. 
M. A. BELTRÁN S. y cols.

Incisional hernia: Open techniques. World J Surg. 2005;29:1066-72.

14. Kurzer M, Kark A, Selouk S, Belsham P. Open mesh repair of incisional hernia using a sublay technique: Long-term follow-up. World J Surg. 2008;32:31-6.

15. Mehrabi M, Jangjoo A, Tavoosi H, Kahrom M, Kahrom H. Long-term outcome of Rives-Stoppa technique in complex ventral incisional hernia. World J Surg. 2010;34:1696-701.

16. Beltrán MA, Burgos CC, Paredes MF, Martínez HG, Larenas RU, Tapia TF, y cols. Resultados y seguimiento alejado de la hernioplastía de Lichtenstein: Aplicación de un instrumento cualitativo y cuantitativo de medición. Rev Chil Cir. 2005;57:320-9.

17. Fränneby U, Gunnarsson U, Wollert S, Sandblom G. Discordance between the patient's and surgeon's perception of complications following hernia surgery. Hernia 2005;9:145-9.

18. Yahchouchy-Chouillard E, Aura T, Picone O, Etienne JC, Fingerhurt A. Incisional hernias - I. Related factors. Dig Surg. 2003;20:3-9.

19. Iqbal CW, Pham TH, Joseph A, Mai J, Thompson GB, Sarr MG. Long-term outcome of 254 complex incisional hernia repairs using the modified Rives-Stoppa technique. World J Surg. 2007;31:2398-404.

20. Sauerland S, Korenkov M, Kleinen T, Amdt M, Paul A. Obesity is a risk factor for recurrence after incisional hernia repair. Hernia 2004;8:42-6.

21. Moreno-Egea A, Aguayo-Albasini JL, Ballester MM, Cases-Baldó MJ. Treatment of incisional hernias adopting an intra-abdominal approach with a new lowdensity composite prosthetic material: Proceed: Our preliminary experience on 50 cases. Surg Lap Endosc \& Percutan Tech. 2009;19:497-500.

22. Langer C, Schaper A, Liersch T, Kulle B, Flosman M, Füzesi L, et al. Prognosis factors in incisional hernia surgery: 25 years of experience. Hernia 2005;9:16-21.

23. Rosenberg J, Burcharth J. Feasibility and outcome after laparoscopic ventral hernia repair using Proceed mesh. Hernia 2008;12:453-6.

24. Burger JW, Lange JF, Halm JA, Kleinrensink GJ, Jeekel H. Incisional hernia: Early complication of abdominal surgery. World J Surg. 2005;29:1608-13.

25. Cengiz Y, Israelsson LA. Incisional hernias in midline incisions: An eight-year follow-up. Hernia 1998;2:1757. 